\title{
Radiology and Imaging Technology
}

\section{Use of Acoustic Radiation Force Impulse Elastography to Discrim- ination Benign and Malignant Masses for Bladder}

\author{
Onur DEDE ${ }^{1 *}$, Memik TEKE ${ }^{2}$, Mansur Daggulli' and Necmettin Penbegül1 ${ }^{1}$ \\ ${ }^{1}$ Department of Urology, Dicle University School of Medicine, Dıyarbakır, Turkey \\ ${ }^{2}$ Department of Radiology, Dicle University School of Medicine, Dıyarbakır, Turkey
}

*Corresponding author: Onur DEDE, Assistant Professor of Urology, Department of Urology, Dicle University School of Medicine, 21280 Diyarbakir, Turkey, Tel: 905057019530 , Fax: +90-4122488523

\begin{abstract}
This study was aimed that the evaluation of tissue elasticity for determination of the characterization of bladder masses. The prospective study was performed between May and September 2014. This study included 39 patients who presented with microscopic or gross hematuria and had primary bladder mass and according to ultrasound or computed tomography results. ARFI elastography was performed by one experienced radiologist then, the shear wave velocity was measured. Two measurements were performed on each bladder mass and average values were evaluated. The mean age of patients was $63.9 \pm 15.2$ and there were 32 male, 7 female patients. The mean diameters of benign and malignant lesions were 1.2 and $4.4 \mathrm{~cm}$, respectively. The mean diameter of bladder mass lesions was $4.87 \pm 2.23$ $\mathrm{cm}$. Transurethral resection of the tumors and histopathologic examination revealed 26 malignant lesions, whereas 13 patients had benign lesions. The mean shear wave velocity (SWV) value of the malign lesions $(3.28 \pm 0.74 \mathrm{~m} /$ $\mathrm{sn})$ was significantly different from benign lesions $1.7 \pm 0.82$ $(p<0.01)$. Our prospective study shows that Acoustic radiation force impulse (ARFI) imaging may be useful for differentiating between benign bladder lesions and malignant bladder tumours. Because ARFI imaging is a non-invasive and low-cost imaging modality, we propose that adding it to conventional sonography may improve accuracy and aid in the differential diagnosis of bladder tumours.
\end{abstract}

\section{Introduction}

The most common malignant tumours of the bladder are urothelial cell carcinoma. Urothelial cell carcinoma is the $11^{\text {th }}$ most common cancer in the World [1]. It is characterized by high recurrence rate, pathological progression and poor survival in advanced metastatic disease. Due to the long follow-up period and associat- ed expenses of disease monitoring it is one of the most expensive cancers to manage [2].

Bladder cancer may be diagnosed incidentally, painless and usually intermittent, macroscopic haematuria is the commonest finding, occurring in about $75 \%$ of patients. Ultrasonography (US), intravenous urography (IVU) and computed tomography (CT) can be easily diagnosed with a mass in bladder. The Standard cystoscopic examinationis performed for detection of mucosal abnormalities leading to bladder biopsy or transurethral resection of the bladder (TURB). European Association of Urology (EAU) guidelines recommend that patients with low-risk Ta tumors should undergo cystoscopy at 3 months, and if findings are negative, subsequent cystoscopy is advised 9 months later, and then annually for 5 years [3]. Although cystoscopy can directly visualize the urethra and the urinary bladder, its limitations are high cost and an invasive procedure [4]. Many investigators have evaluated the diagnostic accuracy of ultrasonography, MR, CT when compared with that of cystoscopy in the first diagnose and follow-up of patients with bladder tumors [5-7]. Ultrasonography is a noninvasive diagnostic procedure and has easy accessibility in the recent past.

Technical advances of ultrasound applications and post-processing developments have enabled the assessment of new aspects in the structural and functional analysis of many different tissue $[8,9]$. The term Acoustic radiation force impulse (ARFI) refers to a range of techniques that attempt to measure issue stiffness, which has long been recognized as a useful indicator of 
disease. The general principle is to image the dissuade formation induced by some sort of applied mechanical stress but ARFI use shear wave velocity. Stiff lesions deform less than their surroundings and are often readily apparent in the resulting elastograms [10]. Because malignant tissues are generally stiffer than benign tissues, this study was aimed that the evaluation of tissue elasticity might be useful for the characterization of bladder masses.

\section{Material and Methods}

A prospective study was performed between May and September 2014. This study was approved by the Institutional Review Board of the University Medical Faculty. This study included 39 patients who presented with microscopic or gross hematuria and had primary bladder mass and according to ultrasound or computed tomography results. The patients who had a bladder mass less than $10 \times 6 \mathrm{~mm}$, operation before for bladder mass were exclude the study. ARFI elastography was performed with the same transducer by one experienced radiologist with 10 years of clinical experience in abdominal US imaging and 2 years of experience in elastography using an Acuson S2000TM scanner with a curvilinear transducer array operating at $4 \mathrm{MHz}(4 \mathrm{C} 1$, Siemens Medical Solutions, Mountain View, CA, USA) ARFI quantification was performed after B-mode images of the bladder lesions were obtained in the conventional manner. In each patient, 4 valid SWV measurements were performed on the bladder mass, and the mean values were calculated with the results being expressed in meters/second $(\mathrm{m} / \mathrm{s})$. Then, the shear wave velocity (SWV), expressed in meters per second) was measured in a central window of $3 \mathrm{~mm}$ axial by $2 \mathrm{~mm}$ width with in the ROI that was graphically displayed at $1 \mathrm{~cm}$ axial by $6 \mathrm{~mm}$ width. The patients underwent both B-mode and ARFI elastographic examinations in the supine position. The median ARFI value was used as a representative value of the three ARFI values of the bladder mass.

The size of each mass was measured with B-mode US. Masses were evaluated quantitatively by ARFI imaging. Imaging findings were obtained by monitoring the patients and by histopathological evaluations after transurethral resection of bladder tumor (TURbt). Two measurements were performed on each bladder mass and average values were evaluated.

\section{Statistical analysis}

Statistical analysis was performed using Statistical Package for Social Sciences (SPSS) 11.5 software (SPSS Inc., Chicago, IL, USA). All the data were presented as mean \pm SD. The distribution of the data was evaluated by the Kolmogorov-Smirnov test. The comparisons of the groups were performed using independent sample t-tests. A p-value of less than 0.05 was considered statistically significant.

\section{Results}

The mean age of patients was $63.9 \pm 15.2$ and there were 32 male, 7 female patients. The mean diameters of benign and malignant lesions were 12 and $44 \mathrm{~mm}$, respectively. The mean diameter of bladder mass lesions were $4.87 \pm 2.23 \mathrm{~cm}$. Transurethral resection of the tumors and histopathologic examination revealed 26 malignant lesions, whereas 13 patients had benign lesions. Of the malignant lesions, 16 were in transitional cell type and 10 were squamous cell bladder cancer. The benign lesions were chronic inflammation for 7 patients, granulomatous cystitis for 3 patients, nephrogenic adenoma for 3 patients. Patients data was summarized Table 1.

The mean SWV value of the malign lesions (3.28 \pm $0.74 \mathrm{~m} / \mathrm{sn}$ ) was significantly different from benign lesions $1.7 \pm 0.82(p<0.01)$. There was not a significant difference between the SWV values of malignant lesions subgroups. There was a malign lesion ARFI image and SWV value in Figure 1 and a benign lesion ARFI image and SWV value in Figure 2.

\section{Discussion}

Bladder cancer is the $7^{\text {th }}$ most common cancer in men and the $17^{\text {th }}$ most common in women worldwide. It can often be found early because it causes haematuria or other urinary symptoms. Many types of imaging have been used in recent years to achieve to differentiate between malignant tumors and benign lesions or clinical staging for the optimum treatment of patients

Table 1: Patients data was summarized.

\begin{tabular}{|c|c|c|c|}
\hline Age & $63.9 \pm 15.2$ & & \\
\hline \multicolumn{4}{|l|}{ Gender } \\
\hline Male & 32 & & \\
\hline Female & 7 & & \\
\hline Diameter of bladder mass $(\mathrm{cm})$ & $4.87 \pm 2.23$ & & \\
\hline Histopathologic examination & 39 & Size mean $(\mathrm{cm})$ & SWV mean \pm SD $(\mathrm{m} / \mathrm{s})$ \\
\hline Malignant lesions subgroups & 26 & & \\
\hline Transitional cell type & 16 & $4.14 \pm 1.15$ & $3.31 \pm 0.79$ \\
\hline Squamous cell & 10 & $3.74 \pm 0.5$ & $3.04 \pm 0.82$ \\
\hline Benign lesions subgroups & 13 & & \\
\hline Chronic inflammation & 7 & $5.74 \pm 2.39$ & $1.52 \pm 0.21$ \\
\hline Granulomatous cystitis & 3 & $4.75 \pm 1.67$ & $1.91 \pm 0.9$ \\
\hline Nephrogenic adenoma & 3 & $5.74 \pm 3.15$ & $1.83 \pm 1.2$ \\
\hline
\end{tabular}




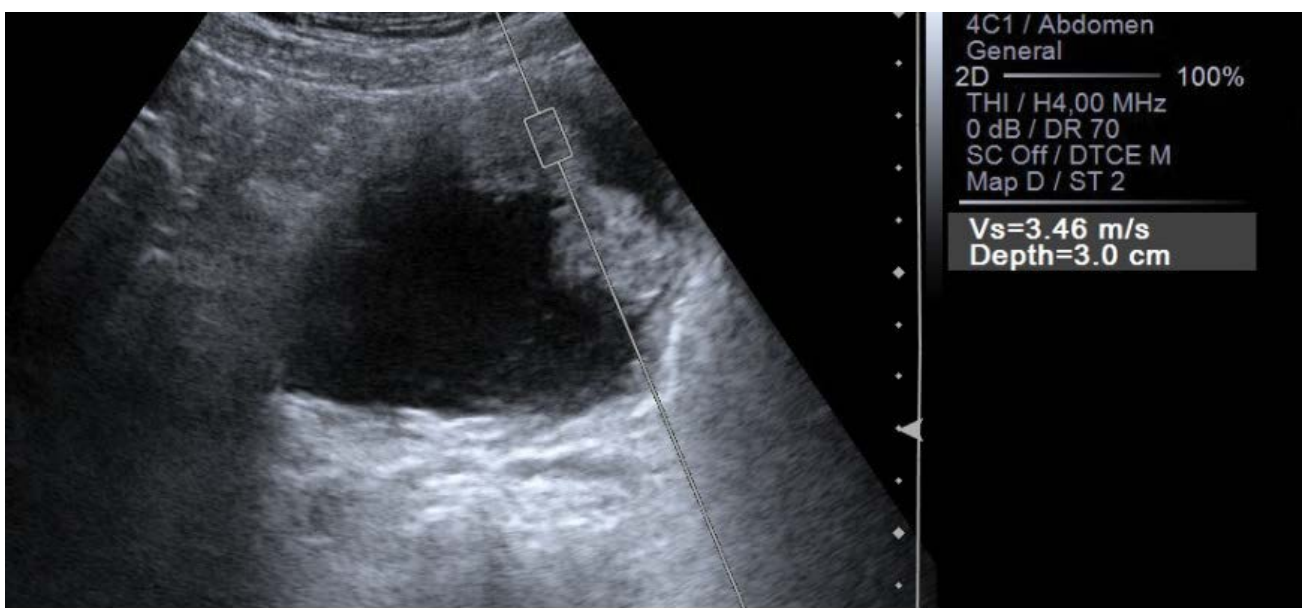

Figure 1: Shear wave velocity (SWV) measurements of the renal tumours were revealed by placing the region of interest (ROI) in the ventral margin of the tumour. a SWV measurement of a histopathologically confirmed clear cell carcinoma in the right kidney of a 45-year-old male patient. b SWV measurement of a histopathologically confirmed chromophobe cell renal carcinoma in the left kidney of a 32-year-old female patient.

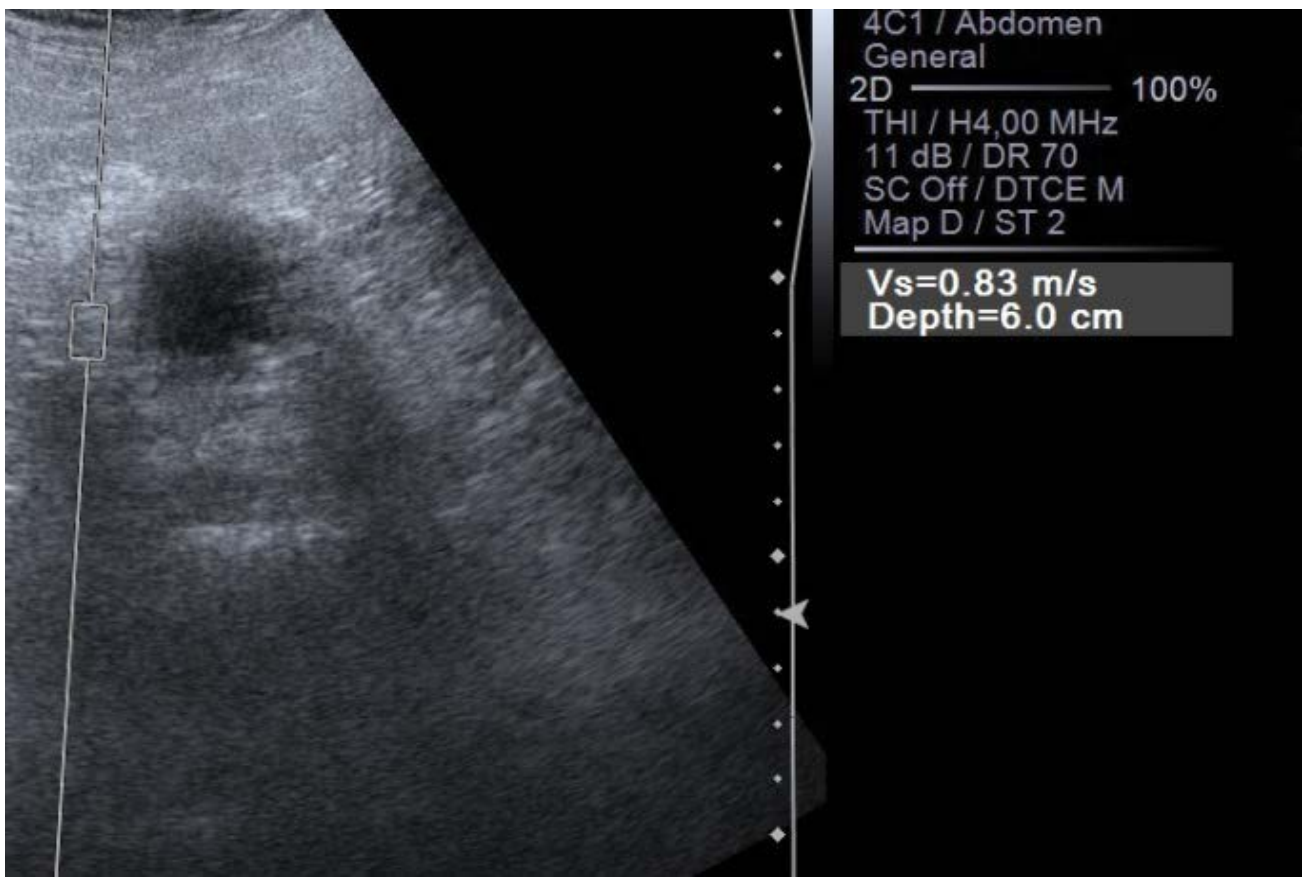

Figure 2: Benign lesion ARFI image and SWV value.

with urothelial carcinoma as intravenous urography (IVU), CT, MR and US. IVU can be give some information especially upper urinary tract and tumour muscle invasion, but particularly in muscle-invasive tumours of the bladder, CT urography gives more information than IVU $[11,12]$. US permits characterization of renal masses, detection of hydronephrosis, and visualization of intraluminal masses in the bladder. It is as accurate as IVU for diagnosis of upper tract urothelial carcinomas [11]. Ultrasound is a useful tool for detection of mass in patients with haematuria. However, it cannot exclude the presence of upper tract urothelial carcinoma and cannot replace CT urography. The diagnosis of carcinoma in situ cannot be made with imaging methods [13].

In comparison to other imaging methods, US is a painless, noninvasive, practical, and radiation-free pro- cedure that requires minimal preparation [11]. Previous studies reported that approximately $80 \%$ of bladder cancer were accurately diagnosed by ultrasonography [5].

Recently, stiffness imaging techniques that measure the shear wave velocity in liver tissues and US-based applied in clinical studies to investigate intra-abdominal tissues [14]. In recent years, this technique has been successfully applied to lesions of the breast, prostate, pancreas, lymph nodes, thyroid gland, testes, renal tumours and liver [8,9]. However, to our knowledge, few studies in which ARFI imaging has been used to evaluate have been previously reported in the medical literature. Our study shows that the measurement of SWV elasticity offers a useful method for the differentiation between benign bladder lesions and malignant bladder le- 
sions. In this study was achieved significant higher SWV values for malignant tumours than benign lesions. It is very use full for discriminate benign malign lesions for bladder with noninvasive technique.

In conclusion, although not yet established for routine clinical use, US elastography is a promising adjunctive modality for evaluating bladder lesions. Our prospective study shows that ARFI imaging may be useful for differentiating between benign bladder lesions and malignant bladder tumours. Because ARFI imaging is a non-invasive and low-cost imaging modality, we propose that adding it to conventional sonography may improve accuracy and aid in the differential diagnosis of bladder tumours.

\section{Conflict of Interest}

No potential conflict of interest relevant to this article was reported.

\section{References}

1. Ferlay J, Shin HR, Bray F, Forman D, Mathers C, et al (2010) Estimates of worldwide burden of cancer in 2008: GLOBOCAN 2008. Int J Cancer 127: 2893-2917.

2. Donat SM (2003) Evaluation and follow-up strategies for superficial bladder cancer. Urol Clin North Am 30: 765-776.

3. Babjuk M, Burger M, Zigeuner R, Shariat SF, van Rhijn BW, et al. (2013) EAU guidelines on non-muscle-invasive urothelial carcinoma of the bladder: update 2013. European urology 64: 639-653.

4. Burke DM, Shackley DC, O'Reilly PH (2002) The community-based morbidity of flexible cystoscopy. BJU Int 89: 347349.
5. Pavlica P, Gaudiano C, Barozzi L (2004) Sonography of the bladder. World J Urol 22: 328-334.

6. Rosenkilde Olsen $\mathrm{P}$, Jorgensen PM, Roed-Petersen $\mathrm{K}$, Iversen HG, Iversen Hansen R, et al. (1985) Control for recurrences of urinary bladder tumours by transabdominal ultrasonic scanning. Scandinavian Journal of Urology and Nephrology 19: 105-107.

7. Daggulli M, Onur MR, Firdolas F, Onur R, Kocakoc E, et al. (2011) Role of diffusion MRI and apparent diffusion coefficient measurement in the diagnosis, staging and pathological classification of bladder tumors. Urol Int 87: 346-352.

8. Goya C, Daggulli M, Hamidi C, Yavuz A, Hattapoglu S, et al. (2015) The role of quantitative measurement by acoustic radiation force impulse imaging in differentiating benign renal lesions from malignant renal tumours. Radiol Med 120: 296-303.

9. Dede O, Teke M, Daggulli M, Utangac M, Bas O, et al. (2016) Elastography to assess the effect of varicoceles on testes: a prospective controlled study. Andrologia 48: 257-261.

10. Ophir J, Cespedes I, Ponnekanti H, Yazdi Y, Li X (1991) Elastography: a quantitative method for imaging the elasticity of biological tissues. Ultrasonic Imaging 13: 111-134.

11. Goessl C, Knispel HH, Miller K, Klan R (1997) Is routine excretory urography necessary at first diagnosis of bladder cancer? J Urol 157: 480-481.

12. Nolte-Ernsting C, Cowan N (2006) Understanding multislice CT urography techniques: Many roads lead to Rome. Eur Radiol 16: 2670-2686.

13. Tetu B (2009) Diagnosis of urothelial carcinoma from urine. Mod Pathol 2: S53-S59.

14. Nightingale K, Soo MS, Nightingale R, Trahey G (2002) Acoustic radiation force impulse imaging: in vivo demonstration of clinical feasibility. Ultrasound Med Biol 28: 227235. 